\title{
Procalcitonina sérica como biomarcador diagnóstico de derrame paraneumónico o empiema
}

\author{
Ángel Emmanuel Vega-Sánchez, José Luis Che-Morales, Gary Kosai Vargas-Mendoza, \\ Danielle Aimee Manjarrez-Martín, Arturo Cortés-Telles $\bowtie$
}

Hospital Regional de Alta Especialidad de la Península de Yucatán. Mérida, Yucatán.

Trabajo recibido: 12-X-2016; aceptado: 16-XII-2016

\begin{abstract}
RESUMEN. Antecedentes: La relevancia del derrame paraneumónico (DPN) y empiema, radica en su morbimortalidad y tendencia ascendente. Recién ha tomado interés la exploración de nuevos biomarcadores capaces de incidir en su desenlace. La procalcitonina (PCT) es un sólido marcador de infección sistémica; sin embargo, son escasos los trabajos que describen su comportamiento en DPN y empiema. Este estudio exploró si la PCT tiene un papel en el abordaje diagnóstico del derrame pleural unilateral de causa infecciosa manifestado como DPN y/o empiema. Material y métodos: Diseñamos un estudio clínico observacional prospectivo que incluyó a pacientes ingresados a nuestra institución con derrame pleural unilateral por un período de 18 meses. Se analizó el contraste del valor absoluto de PCT sérica en dos grupos de estudio y la probabilidad de predecir infección definida como DPN y/o empiema. Resultados: Se incluyeron 49 pacientes divididos en dos grupos (infeccioso vs. no infeccioso). Al ingreso, el valor de PCT sérica fue diferente entre ambos grupos (1.42 ng $/ \mathrm{mL} v s .0 .83 \mathrm{ng} / \mathrm{mL}$ ). El análisis de regresión identificó que PCT sérica tuvo adecuada asociación con infección pleural. El valor de PCT que mejor discriminó este hallazgo fue $\geq 2$ ng/mL, OR 4.1 (IC 95\% 1.1-15.3; p = 0.038). Conclusiones: Procalcitonina es un biomarcador sérico útil en el diagnóstico de infección pleural. Sin embargo, deben realizarse más estudios para comprobar nuestros resultados.
\end{abstract}

Palabras clave: Biomarcadores, derrame paraneumónico, neumonía, procalcitonina.

ABSTRACT. Background: The relevance of parapneumonic effusion (DPN) and empyema lies in it's morbidity and mortality and the upward trend. It has recently taken interest in exploring new biomarkers able to influence its outcome. Procalcitonin is a solid marker of systemic infection, however, few studies describe the role in DPN and empyema. The current study analyzed if procalcitonin has a role in the approach of unilateral pleural effusion such as parapneumonic pleural effusion and/or empyema. Material and methods: We designed a prospective observational clinical study involving patients admitted to our institution with unilateral pleural effusion for a period of 18 months. The contrast of the absolute value of serum PCT in 2 groups of study and the ability to discriminate infection such as parapneumonic pleural effusion and/ or empyema was analyzed. Results: 49 patients divided into 2 groups (infectious vs. noninfectious) were included. At admission, the value of serum PCT was different between the two groups (1.42 ng/mL vs. $0.83 \mathrm{ng} / \mathrm{mL})$. Regression analysis identified that serum PCT had adequate association with pleural infection. The PCT value that best discriminated this finding was $\geq 2 \mathrm{ng} / \mathrm{mL}, \mathrm{OR} 4.1(95 \% \mathrm{Cl} 1.1-15.3 ; \mathrm{p}=0.038)$. Conclusions: Procalcitonin is a useful serum biomarker in the diagnosis of pleural infection. However, more studies should be performed to reinforce our results.

Key words: Biomarkers, parapneumonic effusion, pneumonia, procalcitonin.

\section{INTRODUCCIÓN}

El derrame paraneumónico (DPN) es el acumulo de líquido en el espacio pleural que ocurre como consecuencia de un proceso neumónico. Por otro lado, la presencia de pus en el espacio pleural se define como empiema. ${ }^{1}$ La frecuencia de estas entidades clínicas se ha duplicado en la última década, de 7.6 a $14.9 \%{ }^{2}$ Cabe recordar, $40-60 \%$ de las neumonías pueden desarrollar DPN; de éstos, entre 10 y $20 \%$ evolucionan hacia un empiema. ${ }^{3,4}$ A pesar de los avances en el área se mantiene como la principal causa de muerte por infección en el mundo. ${ }^{5-7}$ Revisiones sistematizadas que abordan las infecciones en el espacio pleural enfatizan la necesidad de más estudios que brinden información sobre evolución, diagnóstico temprano y desenlace de la enfermedad con la intención de proporcionar un manejo más efectivo. ${ }^{8}$

La procalcitonina (PCT) ha demostrado tener una relación causal con enfermedades sistémicas graves de etiología bacteriana. ${ }^{9} \mathrm{El}$ valor sérico que sugiere este origen es igual o mayor que $0.5 \mathrm{ng} / \mathrm{mL}$ (sensibilidad $77 \%$, especificidad $79 \%$, valor de predicción positiva $83 \%$, valor de predicción negativa $70 \%) .{ }^{10,11} \mathrm{El}$ 
comportamiento del biomarcador es interesante, dado que se caracteriza por una rápida elevación en suero durante la etapa aguda de la infección seguido de un descenso acelerado una vez que se controla la causa precipitante..$^{12}$ Con base en nuestro conocimiento, PCT ha sido empleada en el diagnóstico de infecciones extratorácicas que requieren intervención quirúrgica; ${ }^{13-15}$ asimismo, en la identificación oportuna de infecciones postquirúrgicas. ${ }^{16,17}$

La exploración del rendimiento diagnóstico de PCT sérica en patología pleural de causa infecciosa ha otorgado resultados contrastantes. Algunos informes no han encontrado diferencias en el valor sérico de PCT en tres grupos de estudio (DPN, exudados no bacterianos y trasudados).$^{18}$ Estudios más recientes han explorado valores de PCT para identificar causas infecciosas del derrame pleural (DP)..$^{19}$ Entre las limitantes de los estudios se encuentra la falta de análisis en suero, reproducibilidad de los hallazgos y la variación del rendimiento diagnóstico entre poblaciones. Por tal motivo, el objetivo principal del presente estudio es analizar el comportamiento de PCT sérica al ser evaluada de forma sistematizada en un grupo de pacientes mexicanos confirmados con DPN.

\section{MATERIAL Y MÉTODOS}

\section{Pacientes}

Es un estudio clínico observacional prospectivo que incluyó de manera consecutiva pacientes mayores de 18 años con diagnóstico de DP unilateral. Al finalizar la evaluación inicial fueron divididos en dos grupos de estudio. Primer grupo, diagnóstico de DPN y/o empiema con base en la definición estandarizada utilizada por las sociedades nacionales e internacionales. ${ }^{1}$ Por otro lado, el segundo grupo incluyó pacientes con DP que al finalizar el proceso de evaluación inicial fueran exudados de causa no infecciosa.

\section{Procedimiento}

Previa firma de consentimiento informado al ingreso en el Servicio de Admisión Continua de nuestro hospital, se realizó una toracocentesis diagnóstica extrayendo $110 \mathrm{~mL}$ distribuidos de la siguiente manera: $20 \mathrm{~mL}$ para análisis citoquímico convencional en jeringa heparinizada, $20 \mathrm{~mL}$ para cultivo microbiológico, $10 \mathrm{~mL}$ para análisis del estado ácido-base en jeringa heparinizada y $60 \mathrm{~mL}$ para citológico en combinación con $60 \mathrm{~mL}$ de alcohol al $70 \%$. De manera simultánea se obtuvo una muestra de sangre periférica para la cuantificación específica de PCT, PCR, biometría hemática, pruebas de funcionamiento hepático que incluyera cuantificación sérica de proteínas y nivel sérico de deshidrogenasa láctica. La cuantificación de PCT sérica se realizó en un equipo mini Vidas ${ }^{\circledR}$ B.R.A.H.M.S. con rango de medición que oscilan entre 0.05 a 200 ng/dL por técnica ELFA (Enzyme Linked Fuorecescent Assay). La PCR fue analizada con un analizador cobas c311 por técnica de inmunoturbodimetría con rangos de medición que oscilan entre 0.3 a $350 \mathrm{mg} / \mathrm{L}$. Fueron excluidos los casos cuyo abordaje inicial del DP se llevó a cabo en otra institución y que por alguna circunstancia habían sido referidos a nuestro hospital para continuar su manejo; pacientes que no tenían cuantificado PCT al momento del ingreso, presencia simultánea de dos sitios de infección y, particularmente, en el caso del grupo de comparación, evidencia de infección en un sitio diferente al espacio pleural (orina, bacteremia, etc.). Asimismo, se excluyeron trasudados y exudados secundarios a tuberculosis pleural.

El estudio fue aprobado por los comités de Investigación y Ética en Investigación de nuestro hospital con el número de registro 2014-023.

\section{Análisis estadístico}

Las variables continuas se presentan como medianas con rango intercuartílico (RIC); por otro lado, las variables categóricas como frecuencias con porcentajes. El contraste de grupos se realizó de la siguiente manera: las variables continuas mediante la prueba rangos sumados de Wilcoxon. En tanto, las variables categóricas mediante la prueba de $\chi^{2}$ o la prueba exacta de Fisher según fuera el caso. Se analizó la certeza de PCT sérica en el diagnóstico de infección pleural con la construcción de una curva ROC, a partir de ésta, se obtuvo el valor del área bajo la curva (AUC) con la mejor sensibilidad y especificidad, LR + y LR-; finalmente, para conocer la fuerza de asociación entre la variable central del estudio con la presencia de DPN y/o empiema se realizó un análisis de regresión logística univariado con ajuste de múltiples confusores. Los resultados se expresan como razón de momios (OR) con sus correspondientes intervalos de confianza (IC) al 95\%. Se consideró estadísticamente significativo un valor de $p$ menor al 5\% bimarginal. Para el análisis se empleó el paquete estadístico STATA versión 12 (StataCorp LP, Collage Station, Tx).

\section{RESULTADOS}

El presente estudio incluye un total de 49 pacientes, 26 casos con diagnóstico de infección en el espacio pleural y 23 casos de etiología no infecciosa. La mediana de 
edad no fue diferente entre los grupos de estudio, 60 años (RIC 45-66) vs. 55 años (RIC 36-69), $p=0.568$. Por otro lado, entre los antecedentes generales de importancia, la existencia de mala higiene oral fue mayor en los casos con infección del espacio pleural (65\% vs. $35 \%, p=0.046$ ). El resto de las características de la población se presentan en la tabla 1.

Los niveles séricos de PCT, PCR y las características del líquido pleural se exponen en la tabla 2. Cabe destacar, la frecuencia de casos con PCT sérica igual o mayor que $0.5 \mathrm{ng} / \mathrm{mL}$ fue superior en el grupo de infección del espacio pleural ( $88 \%$ vs. $48 \%$, $p=0.002)$; la diferencia en valores absolutos entre los grupos tuvo un comportamiento con tendencia significativa hacia los casos con infección (1.42 ng/ $\mathrm{mL}$ vs. $0.83 \mathrm{ng} / \mathrm{mL}, \mathrm{p}=0.076$ ); asimismo, el contraste del valor absoluto de PCR sérica fue notablemente diferente entre ambos grupos (149 mg/dL vs. $75 \mathrm{mg} /$ $\mathrm{dL}, \mathrm{p}<0.001$ ).

\section{Características microbiológicas}

El microorganismo que se aisló con mayor frecuencia fue E. coli (22\%), seguido del S. pneumoniae con el $18 \%$. En la tabla 3 se resumen los aislamientos.

El tiempo de estancia hospitalaria fue diferente entre ambos grupos, la mediana del tiempo fue 19 días (RIC 12-28) vs. 8 días (RIC 5-20), respectivamente para casos con infección y casos no infecciosos ( $p$ $=0.033)$.

\section{Análisis de regresión logística univariado y multivariado}

Se llevaron a cabo múltiples ensayos para conocer la posibilidad de causa-efecto entre dichas variables considerando la presencia de infección (DPN y/o empiema) como variable dependiente. Cuando PCT es positiva, a partir de $0.5 \mathrm{ng} / \mathrm{mL}$, el OR para infección del líquido pleural fue de 8.4 (IC 95\% 2.0-35.8, p = 0.004) independiente de edad, género y comorbilidades (DM2, historia de cáncer, enfermedades inmunológicas). El valor de PCT en suero que tuvo la mejor certeza diagnóstica para identificar la presencia de infección pleural fue igual o mayor que $2 \mathrm{ng} / \mathrm{mL}$ [AUC 0.64 (IC 95\%, 0.520.77)]. La sensibilidad, especificidad, LR (+) y LR (-) fue de $46 \%, 82 \%, 2.65$ y 0.65, respectivamente. Además, al utilizar el valor como variable dicotómica, persiste como buen indicador para el desenlace primario (infección pleural) con un OR de 4.1 (IC 95\%, 1.1-15.3, p = 0.038), más aún, se mantiene independiente de edad, género y comorbilidades (tabla 4).

También se exploró la capacidad diagnóstica de la PCR sérica para documentar el mismo objetivo (infección pleural). Encontramos que el valor de $143 \mathrm{mg} / \mathrm{dL}$ confiere un OR de 15 (IC 95\% 3.4-65.4, p < 0.001) para dicho diagnóstico. El AUC fue de 0.79 (0.66-0.92) con una sensibilidad, especificidad, LR (+) y LR (-) del 69, $87 \%, 5.31$ y 0.35 , respectivamente.

Se registraron tres decesos, todos fueron en el grupo de causa infecciosa (11.5\%). El motivo, progresión

Tabla 1. Características generales de la población incluida $(n=49)$.

\begin{tabular}{|c|c|c|c|}
\hline Variable & Etiología infecciosa $(n=26)$ & Etiología no infecciosa $(n=23)$ & Valor de $p$ \\
\hline Edad (años) & $60(45-66)$ & $55(36-69)$ & 0.568 \\
\hline Género masculino & $20(77 \%)$ & $12(52 \%)$ & 0.080 \\
\hline Historia de tabaquismo & $12(46 \%)$ & $10(43 \%)$ & 0.851 \\
\hline \multicolumn{4}{|l|}{ Comorbilidades } \\
\hline Diabetes mellitus 2 & $15(58 \%)$ & $8(35 \%)$ & 0.154 \\
\hline Oncológico & $2(15 \%)$ & $5(22 \%)$ & 0.230 \\
\hline Inmunológico & $1(4 \%)$ & $3(13 \%)$ & 0.330 \\
\hline Mala higiene oral & $17(65 \%)$ & $8(35 \%)$ & 0.046 \\
\hline Evolución del padecimiento (días) & $15(8-20)$ & $15(7-60)$ & 0.864 \\
\hline \multicolumn{4}{|l|}{ Síntomas } \\
\hline Tos & $23(88 \%)$ & $17(74 \%)$ & 0.273 \\
\hline Disnea & $23(88 \%)$ & $21(91 \%)$ & 0.743 \\
\hline Dolor torácico & $23(88 \%)$ & 10 (44\%) & 0.002 \\
\hline Fiebre & 23 (88\%) & 5 (22\%) & $<0.001$ \\
\hline Expectoración & 21 (81\%) & 7 (30\%) & 0.001 \\
\hline Hiporexia & $16(62 \%)$ & 13 (56\%) & 0.777 \\
\hline Pérdida de peso & $14(54 \%)$ & $14(61 \%)$ & 0.774 \\
\hline
\end{tabular}

Nota: los valores se presentan como medianas con intervalo intercuartílico y frecuencias con porcentajes. 
Tabla 2. Características serológicas y del líquido pleural $(n=49)$.

\begin{tabular}{lccr}
\hline Variable & Etiología infecciosa $(\mathrm{n}=26)$ & Etiología no infecciosa $(\mathrm{n}=23)$ & Valor de $\mathrm{p}$ \\
\hline Leucocitos totales en suero & $17.5(14.3-22.3)$ & $7.9(6.5-12.4)$ & $<0.001$ \\
PCT mayor que 0.5 $\mathrm{ng} / \mathrm{mL}$ & $23(88 \%)$ & $11(48 \%)$ & 0.002 \\
Nivel de PCT sérica $(\mathrm{ng} / \mathrm{mL})$ & $1.42(0.77-2.8)$ & $0.83(0.4-1.7)$ & 0.076 \\
Nivel de PCR sérica $(\mathrm{mg} / \mathrm{mL})$ & $149(122-180)$ & $75(30-128)$ & $<0.001$ \\
Características del líquido pleural & & & \\
pH & $7.18(6.85-7.38)$ & $7.42(7.35-7.45)$ & 0.001 \\
Proteínas (g/dL) & $4.2(3.1-4.7)$ & $4.2(20.9-5)$ & 0.897 \\
Relación proteínas Lp/S & $0.78(0.65-1.02)$ & $0.65(0.55-0.77)$ & 0.203 \\
DHL (UI/L) & $2,871(1,157-10,798)$ & $444(212-853)$ & $<0.001$ \\
Relación DHL Lp/S (UI/L) & $4(1.8-8.2)$ & $0.84(0.60-1.80)$ & 0.001 \\
Glucosa (mg/dL) & $45(3.6-98)$ & $96(78-146)$ & 0.016 \\
Leucocitos (x 10 cel/mcl) & $7.9(2.1-13.8)$ & $0.7(0.44-3.3)$ & 0.002 \\
PMN (\%) & $85(70-94)$ & $20(9-50)$ & $<0.001$ \\
MN (\%) & $15(6-30)$ & $80(50-91)$ & $<001$ \\
Índice leucocitos LP/S & $0.51(0.14-0.97)$ & $0.1(0.06-0.24)$ & 0.028 \\
ADA (UI/L) & $14(7-16.5)$ & $10(5-18)$ & 0.877 \\
Triglicéridos (mg/dL) & $43(27-54)$ & $46(28-79)$ & 0.394 \\
\hline
\end{tabular}

Abreviaturas: $\mathrm{PCT}=$ procalcitonina; $\mathrm{PCR}=$ proteína $\mathrm{C}$ reactiva; $\mathrm{DHL}=$ deshidrogenasa láctica; $\mathrm{DHL} \mathrm{Lp} / \mathrm{s}=$ índice líquido pleural/suero; $\mathrm{PMN}=$ polimorfonucleares; $\mathrm{MN}=$ mononucleares; $\mathrm{ADA}=$ adenosindeaminasa .

Nota: los valores se presentan como medianas con intervalo intercuartílico y frecuencias con porcentajes.

Tabla 3. Aislamientos microbianos en líquido pleural.

\begin{tabular}{lcc}
\hline Microorganismo & $\begin{array}{c}\text { Frecuencia } \\
\text { de casos }\end{array}$ & Porcentaje \\
\hline Escherichia coli & 6 & 22.2 \\
Streptococcus pneumoniae & 5 & 18.5 \\
Pseudomonas aeruginosa & 4 & 14.8 \\
Klebsiella pneumoniae & 4 & 14.8 \\
Staphylococcus aureus & 3 & 11.1 \\
Enterococcus faecalis & 3 & 11.1 \\
Streptococcus beta hemolítico & 1 & 3.7 \\
Candida & 1 & 3.7 \\
\hline
\end{tabular}

de la enfermedad hasta choque séptico y disfunción multiorgánica.

\section{DISCUSIÓN}

Con base en nuestro conocimiento, éste es el primer estudio realizado en la población mexicana donde se explora el rendimiento diagnóstico de PCT como biomarcador sérico que apoya la existencia de infección del espacio pleural. Los hallazgos más relevantes se circunscriben a continuación: 1) la positividad de PCT en suero (definido con un valor igual o mayor a $0.5 \mathrm{ng} /$ $\mathrm{mL}$ ) es útil en la identificación de DP unilateral de causa infecciosa; 2) el mejor rendimiento diagnóstico de PCT sérica se observó con un valor absoluto igual o mayor a
Tabla 4. Análisis de regresión logística multivariado con base en la existencia de infección pleural.

\begin{tabular}{lccc}
\hline Variable & OR & IC 95\% & Valor de p \\
\hline $\begin{array}{l}\text { Morbilidades } \\
\quad \text { Mala higiene oral }\end{array}$ & 3.5 & $1.09-11.5$ & 0.035 \\
$\begin{array}{l}\text { Bioquímicos } \\
\quad \text { PCT positiva (mayor }\end{array}$ & 8.4 & $2.0-35.8$ & 0.004 \\
$\quad \begin{array}{l}\text { a } 0.5 \mathrm{ng} / \mathrm{mL} \text { ) } \\
\text { PCT mayor o igual }\end{array}$ & 4.1 & $1.1-15.3$ & 0.038 \\
$\begin{array}{l}\text { a } 2 \mathrm{ng} / \mathrm{mL} \\
\text { PCR mayor o igual }\end{array}$ & 15 & $3.4-65.4$ & $<0.001$ \\
$\quad$\begin{tabular}{l} 
a $143 \mathrm{mg} / \mathrm{mL}$ \\
\hline
\end{tabular}
\end{tabular}

Abreviaturas: $\mathrm{PCT}=$ procalcitonina; $\mathrm{PCR}=$ proteína $\mathrm{C}$ reactiva . Nota: el valor se ajustó para las siguientes variables: edad, género, tabaquismo y morbilidades.

$2 \mathrm{ng} / \mathrm{mL}$; 3) por otro lado, PCR sérico es un biomarcador adicional que puede respaldar la identificación de DPN.

Un porcentaje no despreciable de pacientes con neumonía evolucionan hacia DPN y empiema. ${ }^{4}$ Como se ha señalado previamente, esta entidad clínica representa un problema de salud por sus complicaciones. Así, diferentes sociedades en el mundo enfatizan la necesidad de explorar otros auxiliares en el diagnóstico que permitan reconocer de forma oportuna estos casos. Derivado de estas recomendaciones, uno de los primeros informes sobre el rendimiento de PCT en 
DPN fue publicado por Lin et al., quienes describen el comportamiento que tiene el biomarcador en el espacio pleural. De manera interesante el rendimiento fue notable para el diagnóstico de derrames pleurales infectados, tanto DPN como empiema (0.834, IC 95\% 0.74-0.915 y 0.752 , IC 95\% 0.618-0.820, respectivamente). ${ }^{20}$ Dos años después, en el 2011, Wang et al. evaluaron un escenario similar, no obstante, la adición de su trabajo al contexto clínico es la incorporación de un valor de referencia para identificar los DPN; el nivel de PCT pleural igual o mayor de $0.18 \mathrm{ng} / \mathrm{dL}$ confirió un AUC de 0.77 (sensibilidad $69.7 \%$ y especificidad $72.1 \%$ ). Derivado de estos hallazgos, exploraron la correlación entre los valores obtenido de PCT pleural con el valor sérico encontrando una fuerza de asociación muy sólida $(r 2=0.967, p<0.001)$. La limitante de este análisis es la muestra (PCT sérica cuantificada únicamente en 16 pacientes). ${ }^{21}$

Nuestros hallazgos no son diferentes de lo previamente informado, pero con un mayor número de sujetos analizados. Primero, $88 \%$ de los casos en quienes se confirmó DPN y/o empiema tuvieron un valor de PCT sérica similar al que recomiendan los estándares para definir la existencia de un proceso infeccioso de origen bacteriano (mayor o igual que $0.5 \mathrm{ng} / \mathrm{mL}$ ). ${ }^{11,22,23}$ Más aún, el valor absoluto fue mayor en comparación con exudados de causa no infecciosa (1.42 ng/mL vs. $0.83 \mathrm{ng} / \mathrm{mL})$. El análisis de regresión confirmó que cuando la PCT sérica es positiva, la probabilidad de enfrentar un escenario de DP de causa infecciosa es 8.4 veces superior. Más aún, la curva ROC identificó que un valor igual o mayor que $2 \mathrm{ng} / \mathrm{mL}$ (AUC 0.64, IC 95\%, 0.52-0.77) tiene una aceptable precisión diagnóstica de etiología infecciosa; la fortaleza del hallazgo se mantiene en el ajuste multivariado (OR 4.1, IC 95\% 1.1-15.3; $p=$ 0.038 ) al considerar el valor de corte como variable binomial (mayor o igual a 2 vs. menor de 2).

Luego entonces, podemos señalar que el acumulo de evidencia continúa posicionando a PCT sérica como biomarcador adjunto de infección pleural. La vinculación fisiopatológica ha sido informada en diversos documentos enfatizando que PCT es liberada al torrente sanguíneo en respuesta al estímulo y activación de monocitos como consecuencia del paso de citocinas inflamatorias desde las zonas de infección en el espacio pleural. ${ }^{23,24}$ Ya se ha demostrado que la inflamación y, de forma subsecuente, el desarrollo de engrosamiento pleural se relacionan con un antecedente común, la presencia de citocinas como factor de crecimiento transformante- $\beta 1$, quien a su vez estimula mayor producción y circulación de PCT sérica. De no solucionar este proceso fisiopatológico con intervenciones terapéuticas oportunas puede incidir en secuelas respiratorias que afecten la calidad de vida. ${ }^{24,25}$

Otro factor que parece contribuir con los niveles séricos de PCT es el tipo de bacteria involucrada en la fisiopatología del DPN; recién se ha informado que la PCT tiene mayor elevación sérica cuando la etiología infecciosa es por bacterias Gram negativas en comparación con infecciones por Gram positivas y hongos..$^{26,27}$ Nuestro estudio no profundizó esta asociación, a pesar de ello, $50 \%$ de los aislamientos fueron de bacterias Gram negativas. Los autores consideramos que la presentación de las enfermedades difieren entre poblaciones, es decir, parte de los contrastes encontrados pueden estar relacionados con la población analizada.

Finalmente, no omitimos la descripción de los datos encontrados de PCR sérica. Estudios previos han ratificado el valor diagnóstico de PCR para casos con DP de origen infeccioso. ${ }^{28,29}$ Inclusive, se han sugerido valores de PCR para orientar hacia la gravedad del problema. ${ }^{30}$ Porcel et al., han señalado un valor de PCR sérica igual o mayor que $200 \mathrm{mg} / \mathrm{L}$ para diferenciar DPN simples de complicados (excluyendo empiemas) con una sensibilidad del $58 \%$, especificidad del $81 \%$ y un AUC de 0.67. De manera interesante y recordando el posible papel que tiene la raza en la presentación de enfermedades, nuestro informe refuerza el valor de PCR sérica en el diagnóstico de DPN y/o empiema; más aún, el nivel propuesto en el análisis ROC (143 $\mathrm{mg} / \mathrm{dL}$ ), discrimina con mejor precisión y los valores probabilísticos son superiores a lo informado. Es posible que la combinación de ambos biomarcadores, PCT y PCR, pudieran incrementar la certeza en el diagnóstico y gravedad de estas entidades clínicas.

\section{Limitaciones del estudio}

Entre las limitantes de nuestro estudio podemos mencionar el número de pacientes que conforma el presente informe y el origen unicéntrico, pues contribuyen con la falta de poder para otorgar una validez externa. No obstante, como fortaleza, los pacientes fueron incluidos de forma consecutiva y con base en nuestro conocimiento, además, es el primer informe que incluye población mexicana. Del mismo modo, no se realizó correlación de la PCT sérica con PCT pleural, en función de reforzar la similitud de ambas mediciones en escenarios donde se dificulte el acceso a uno u otro método. Por último, el valor de corte que proponemos tuvo un área bajo la curva de regular a bueno con base en los intervalos sugeridos; sin embargo, es el primer informe en población latina y los valores se asociaron fuertemente con el riesgo de tener infección del espacio pleural. 


\section{CONCLUSIONES}

En el abordaje del derrame pleural unilateral, la medición de PCT sérica mostró tener un buen perfil diagnóstico como biomarcador complementario a las mediciones convencionales cuando se sospecha que la etiología sea infecciosa; más aún, la probabilidad se incrementa notablemente cuando el valor sérico es mayor de $2 \mathrm{ng} / \mathrm{dL}$. Para corroborar e incrementar el valor de estos hallazgos, consideramos que deben realizarse estudios que incluyan un mayor número de pacientes y con similares características poblacionales.

\section{REFERENCIAS}

1. Davies HE, Davies RJ, Davies CW; MBTS Pleural Disease Guideline Group. Management of pleural infection in adults: British Thoracic Society Pleural Disease Guideline 2010. Thorax 2010;65 Suppl 2:ii41-53. doi: 10.1136/ thx.2010.137000.

2. Burgos J, Falcó V, Pahissa A. The increasing incidence of empyema. Curr Opin Pulm Med 2013;19(4):350-356. doi: 10.1097/MCP.0b013e3283606ab5.

3. Porcel JM, Light RW. Parapneumonic pleural effusions and empyema in adults: current practice. Rev Clin Esp 2009;209(10):485-494.

4. Bhatnagar R, Maskell NA. Treatment of complicated pleural effusions in 2013. Clin Chest Med 2013;34(1):4762. doi: 10.1016/j.ccm.2012.11.004.

5. Grijalva CG, Zhu Y, Nuorti JP, Griffin MR. Emergence of parapneumonic empyema in the USA. Thorax 2011;66(8):663-668. doi: 10.1136/thx.2010.156406.

6. Finley C, Clifton J, Fitzgerald JM, Yee J. Empyema: an increasing concern in Canada. Can Respir J 2008;15(2):85-89.

7. Maskell NA, Batt S, Hedley EL, Davies CW, Gillespie $\mathrm{SH}$, Davies RJ. The bacteriology of pleural infection by genetic and standard methods and its mortality significance. Am J Respir Crit Care Med 2006;174(7):817823.

8. Ferreiro L, San José ME, Valdés L. Management of parapneumonic pleural effusion in adults. Arch Bronconeumol 2015;51(12):637-646. doi: 10.1016/j. arbres.2015.01.009.

9. Becker KL, Snider R, Nylen ES. Procalcitonin assay in systemic inflammation, infection, and sepsis: clinical utility and limitations. Crit Care Med 2008;36(3):941952. doi: 10.1097/CCM.0B013E318165BABB.

10. Becker KL, Nylén ES, White JC, Müller B, Snider RH Jr. Clinical review 167: Procalcitonin and the calcitonin gene family of peptides in inflammation, infection, and sepsis: a journey from calcitonin back to its precursors. J Clin Endocrinol Metab 2004;89(4):1512-1525.

11. Riedel S. Procalcitonin and the role of biomarkers in the diagnosis and management of sepsis. Diagn Microbiol Infect Dis 2012;73(3):221-227. doi: 10.1016/j. diagmicrobio.2012.05.002.
12. Schuetz P, Christ-Crain M, Müller B. Procalcitonin and other biomarkers to improve assessment and antibiotic stewardship in infections--hope for hype? Swiss Med Wkly 2009;139(23-24):318-326. doi: smw-12584.

13. Mofidi R, Suttie SA, Patil PV, Ogston S, Parks RW. The value of procalcitonin at predicting the severity of acute pancreatitis and development of infected pancreatic necrosis: systematic review. Surgery 2009;146(1):72-81. doi: 10.1016/j.surg.2009.02.013.

14. Bezmarevic M, Mirkovic D, Soldatovic I, et al. Correlation between procalcitonin and intra-abdominal pressure and their role in prediction of the severity of acute pancreatitis. Pancreatology 2012;12(4):337-343. doi: 10.1016/j.pan.2012.05.007.

15. Maier M, Wutzler S, Lehnert M, et al. Serum procalcitonin levels in patients with multiple injuries including visceral trauma. J Trauma 2009;66(1):243-249. doi: 10.1097/ TA.0b013e31817c966f.

16. Suberviola B, Castellanos-Ortega A, Ballesteros MA, Zurbano F, Naranjo S, Miñambres E. Early identification of infectious complications in lung transplant recipients using procalcitonin. Transpl Infect Dis 2012;14(5):461467. doi: 10.1111/j.1399-3062.2012.00780.x.

17. Sponholz C, Sakr Y, Reinhart K, Brunkhorst F. Diagnostic value and prognostic implications of serum procalcitonin after cardiac surgery: a systematic review of the literature. Crit Care 2006;10(5):R145.

18. Ko YC, Wu WP, Hsu CS, Dai MP, Ou CC, Kao CH. Serum and pleural fluid procalcitonin in predicting bacterial infection in patients with parapneumonic effusion. J Korean Med Sci 2009;24(3):398-402. doi: 10.3346/ jkms.2009.24.3.398.

19. Doğan C, Bilaçeroğlu S, Çirak AK, Özsöz A, Özbek D. Diagnostic value of pleural fluid and serum procalcitonin levels in the diagnosis of parapneumonic pleural effusion. Tuberk Toraks 2013;61(2):103-109.

20. Lin MC, Chen YC, Wu JT, Ko YC, Wang CC. Diagnostic and prognostic values of pleural fluid procalcitonin in parapneumonic pleural effusions. Chest 2009;136(1):205211. doi: 10.1378/chest.08-1134.

21. Wang CY, Hsiao YC, Jerng JS, et al. Diagnostic value of procalcitonin in pleural effusions. Eur J Clin Microbiol Infect Dis 2011;30(3):313-318. doi: 10.1007/s10096-0101082-0.

22. Zou MX, Zhou RR, Wu WJ, Zhang NJ, Liu WE, Fan XG. The use of pleural fluid procalcitonin and C-reactive protein in the diagnosis of parapneumonic pleural effusions: a systemic review and meta-analysis. Am J Emerg Med 2012;30(9):1907-1914. doi: 10.1016/j.ajem.2012.04.004.

23. Meisner M. Update on procalcitonin measurements. Ann Lab Med 2014;34(4):263-273. doi: 10.3343/ alm.2014.34.4.263.

24. Linscheid P, Seboek D, Schaer DJ, Zulewski H, Keller U, Müller B. Expression and secretion of procalcitonin and calcitonin gene-related peptide by adherent monocytes and by macrophage-activated adipocytes. Crit Care Med 2004;32(8):1715-1721.

25. Sasse SA, Jadus MR, Kukes GD. Pleural fluid transforming growth factor-beta 1 correlates with pleural fibrosis 
in experimental empyema. Am J Respir Crit Care Med 2003;168(6):700-705.

26. Guo SY, Zhou Y, Hu QF, Yao J, Wang H. Procalcitonin is a marker of gram-negative bacteremia in patients with sepsis. Am J Med Sci 2015;349(6):499-504. doi: 10.1097/ MAJ.0000000000000477.

27. Meisner M, Tschaikowsky K, Palmaers T, Schmidt J. Comparison of procalcitonin (PCT) and C-reactive protein (CRP) plasma concentrations at different SOFA scores during the course of sepsis and MODS. Crit Care 1999;3(1):45-50.

28. Porcel JM. Pleural fluid biomarkers: beyond the Light criteria. Clin Chest Med 2013;34(1):27-37. doi: 10.1016/j. ccm.2012.11.002.

29. Skouras V, Boultadakis E, Nikoulis D, et al. Prognostic value of C-reactive protein in parapneumonic effusions. Respirology 2012;17(2):308-314. doi: 10.1111/j.14401843.2011.02078.x.
30. Porcel JM, Bielsa S, Esquerda A, Ruiz-González A, Falguera M. Pleural fluid C-reactive protein contributes to the diagnosis and assessment of severity of parapneumonic effusions. Eur J Intern Med 2012;23(5):447-450. doi: 10.1016/j.ejim.2012.03.002.

\section{$\triangle$ Correspondencia:}

Dr. Arturo Cortés-Telles, Laboratorio de Fisiología Pulmonar, Departamento de Neumología y Cirugía de Tórax. Hospital Regional de Alta Especialidad de la Península de Yucatán.

Calle 7 por 20 y 22 Núm. 433, Fraccionamiento Altabrisa, 97130, Mérida, Yucatán, México. Tel: (999) 942-7600, Extensión: 54302 Correo electrónico: dr_morenheim@hotmail.com Los autores declaran no tener conflicto de intereses. 